\title{
Effect of Ion-Neutral Charge Exchange on Radial Electric Fields in the Edge Plasma
}

\author{
D. A. D'Ippolito and J. R. Myra \\ Lodestar Research Corporation, 2400 Central Avenue, Boulder, Colorado 80301
}

September 28, 2001

(submitted to Physics of Plasmas) 


\title{
Effect of Ion-Neutral Charge Exchange on Radial Electric Fields in the Edge Plasma
}

\author{
D. A. D'Ippolito ${ }^{\dagger}$ and J. R. Myra \\ Lodestar Research Corporation, 2400 Central Avenue, Boulder, Colorado 80301
}

\begin{abstract}
A fluid treatment of the perpendicular plasma conductivity is extended to include the effects of arbitrary ion-neutral collision frequency $v$ and large perpendicular drifts. It is shown that ion-neutral friction (charge-exchange) can give a non-monotonic relationship between the non-ambipolar radial current and the radial electric field, leading to a bifurcated plasma equilibrium. This bifurcation may influence the properties of the $\mathrm{L}-\mathrm{H}$ transition in the presence of neutrals. The physical origin of this effect is an interplay between the surface-averaged equilibrium drifts and the small poloidal variations in the pressure and parallel flow around the flux surface generated by the geodesic curvature.
\end{abstract}

†email: $\underline{\text { dasd@lodestar.com }}$ 


\section{Introduction}

Recently, a number of experiments have been devoted to studying the effect of neutral particle influxes on the high-confinement mode (H-mode), ${ }^{1-5}$ and it has been shown that the neutrals can significantly affect the transition from the low to high confinement modes (the L-H transition). The physical mechanism by which neutrals influence this transition has not yet been identified, and it is not known whether the same mechanism is operative in all experiments. Neutral particles have a strong influence on the particle, momentum and energy balance in the edge plasma through processes such as charge exchange and ionization, so there are many possible physical effects. Recent studies indicate that it is the neutral population inside the separatrix, rather than the SOL neutrals, that correlates with the H-mode transition, 3,5 and that the required threshold power scales with the ion-neutral frictional damping rate. ${ }^{3}$ As this friction is typically dominated by ion-neutral charge exchange, in this paper we will use the terms "friction" and "charge exchange" interchangeably.

In one model of the $\mathrm{H}$-mode, ${ }^{6}$ it is assumed that the $\mathrm{L}$ - and $\mathrm{H}$-mode phases correspond to different roots of the plasma ambipolarity constraint at the plasma edge, $J_{r}$ $=\sigma_{\perp} \mathrm{E}_{\mathrm{r}}$. Here, $\mathrm{J}_{\mathrm{r}}$ is the plasma return current induced by a non-ambipolar radial current due to ion orbit loss, turbulence or external probes; $\sigma_{\perp}$ is the perpendicular plasma conductivity depending on neoclassical viscosity and other dissipative processes; and $\mathrm{E}_{\mathrm{r}}$ is the equilibrium radial electric field. If $\mathrm{J}_{\mathrm{r}}$ is non-monotonic, there will be multiple values of $E_{r}$ over some range of currents, and the L-H transition corresponds to a jump from one root to another. There have been several papers on plasma bifurcations due to damping by plasma viscosity alone6-8 or viscosity combined with neutral friction. 9,10 These papers typically use plasma models appropriate to the fully-ionized, relatively high temperature plasma that exists well inside the separatrix of a tokamak. Recently, there has been a growing interest in the properties of the edge plasma just inside the separatrix, stimulated by the realization that the edge strongly affects the core confinement. The edge plasma is quite different from the core in two important respects: the presence of large radial electric fields and their concomitant $\mathbf{E} \times \mathbf{B}$ drifts, and the presence of a significant neutral particle population. In the present paper, we use a collisional fluid model to show that these characteristics can lead to equilibrium bifurcations driven by 
ion-neutral friction alone in addition to those driven by plasma viscosity. To the best of our knowledge, this is a new result which is complementary to the bifurcation analyses already discussed in the literature. Recent experimental evidence suggests that not all observed L-H transitions are related to bifurcations, but we expect that an understanding of ambipolarity will still be fundamental. The new physics discussed in this paper may aid in understanding the effect of neutrals on H-mode properties such as the threshold power for L-H transitions.

If one assumes a toroidally axisymmetric equilibrium, a non-trivial ambipolarity constraint requires that the conservation of toroidal angular momentum be broken by a dissipative mechanism. To focus the discussion on the neutral-dominated regime in this paper, we neglect the effect of plasma viscosity. The perpendicular plasma conductivity $\sigma_{\perp}$ is then completely determined by the ion-neutral friction. The neutrals are treated as poloidally uniform and stationary. (The validity conditions for these approximations are discussed in Sec. II.) We calculate $\sigma_{\perp}$ using a simple model: fluid equations for particle, charge and momentum conservation, supplemented by the parallel component of Ohm's Law. The calculation is carried out for a parameter regime characterized by both strong neutral friction and strong poloidal variation, which may be relevant to the edge plasma near the separatrix in tokamaks. The resulting conductivity is sensitive to the magnetic geometry (through the geodesic curvature and the parallel connection length), to the ionneutral collision frequency $v$, and to the perpendicular drift frequencies.

Key parameters in the present theory are $\alpha \approx \nu \omega_{\mathrm{E}} / \omega_{\|}^{2}$ and $\delta=\lambda / \mathrm{L}_{\|} \approx \omega_{\|} / \nu$. Here, $\omega_{\mathrm{E}}=\mathrm{cE}_{\mathrm{r}} /(\mathrm{aB})$ is the $\mathbf{E} \times \mathbf{B}$ drift frequency, $\omega_{\|}=\mathrm{c}_{\mathrm{S}} / \mathrm{L}_{\|}$is the parallel transit frequency, $\mathrm{L}_{\|} \approx \mathrm{qR}$ is the parallel connection length, $\mathrm{q}$ is the safety factor, a and $\mathrm{R}$ are the minor and major radii, $\lambda=\mathrm{v}_{\mathrm{i}} / v$ is the ion mean free path for ion-neutral charge exchange, $\mathrm{c}_{\mathrm{s}}=\left(\mathrm{T}_{\mathrm{e}} / \mathrm{m}_{\mathrm{i}}\right)^{1 / 2}$ is the ion sound speed, and $\mathrm{v}_{\mathrm{i}}=\left(\mathrm{T}_{\mathrm{i}} / \mathrm{m}_{\mathrm{i}}\right)^{1 / 2}$. Our derivation of $\sigma_{\perp}$ allows arbitrary values of $\alpha$ and $\delta$, but the neglect of plasma viscosity is justified in the PfirschSchlüter regime only when $\delta<v_{\mathrm{ii}} / \omega_{\|}$, where $v_{\mathrm{ii}}$ is the ion-ion collision frequency. The expression for the conductivity obtained in Sec. IV B reduces to the standard results 9,11 that $\sigma_{\perp}=\sigma_{\perp 0}\left(1+2 q^{2}\right)$ as $v \rightarrow 0$ and $\sigma_{\perp}=\sigma_{\perp 0}$ as $v \rightarrow \infty$ with $\sigma_{\perp 0}=\rho v c^{2} / B^{2}$, where $\rho=$ $\mathrm{n}_{\mathrm{i}} \mathrm{m}_{\mathrm{i}}$. When $\alpha$ is of order unity, we show that the function $\sigma_{\perp}(\alpha)$ is not monotonic, implying that an equilibrium bifurcation can be driven by ion-neutral friction in the absence of plasma viscosity. Our treatment does not contradict treatments of neutrals 
based on neoclassical kinetic theory, $9,10,12$ because the assumed parameter regime is different [see Sec. III].

One obtains the following physical picture from this calculation. The poloidal variation of the grad-B drift (or geodesic curvature) produces coupled poloidal modulations in the density, pressure, potential, and parallel flow velocity. This is the manifestation in the present model of the Pfirsch-Schlüter flows of neoclassical theory. The density and pressure modulations are smoothed out by a return flow of ions along the magnetic field and by rapid $\mathbf{E} \times \mathbf{B}$ rotation. The return flow is limited by the ion-neutral friction. The resulting perpendicular conductivity couples the physics of radial electric fields and ion-neutral friction, and it has a local maximum for magnitudes of the radial electric field such that the poloidal drift and parallel flow effects are comparable.

The present work is most closely related in general approach to that of Hassam et

al. ${ }^{13,14}$ on the Stringer spin-up mechanism for H-modes. They used a Braginskii fluid model and a similar ordering of parameters to treat the effect of poloidally-varying particle and momentum sources, but they neglected neutral friction; here we neglect the sources and concentrate on the effect of the friction due to charge exchange.

The plan of this paper is as follows. In Sec. II, we discuss the basic equations and give the derivation of the ambipolarity constraint in general toroidal geometry with no approximations. The particular model used in solving the equations is discussed in Sec. III and the analytic solution is given in Sec. IV. A summary and discussion of the main results is given in Sec. V.

\section{Basic Equations and Ambipolarity Constraint}

The starting point of the calculation is the following set of equilibrium fluid equations for conservation of density, charge and momentum:

$$
\begin{gathered}
\nabla \cdot(\rho \mathbf{u})=0, \\
\nabla \cdot \mathbf{J}=\nabla \cdot\left(\mathbf{J}_{\mathrm{p}}+\mathbf{J}_{\mathrm{s}}\right)=0, \\
\frac{1}{\mathrm{c}} \mathbf{J}_{\mathrm{p}} \times \mathbf{B}=\nabla \mathrm{p}+\nabla \cdot(\rho \mathbf{u u})+\rho v\left(\mathbf{u}-\mathbf{u}_{0}\right),
\end{gathered}
$$


where $\mathbf{J}=\mathbf{J}_{\mathrm{p}}+\mathbf{J}_{\mathrm{s}}$ is the total current density with $\mathbf{J}_{\mathrm{p}}$ and $\mathbf{J}_{\mathrm{s}}$ representing the contributions from the plasma and from external sources (or non-ambipolar processes), $\mathbf{B}=\mathbf{B b}$ is the magnetic field, $\mathrm{p}=\mathrm{p}_{\mathrm{e}}+\mathrm{p}_{\mathrm{i}}$ is the plasma pressure, $\mathrm{p}_{\mathrm{j}}=\mathrm{n}_{\mathrm{j}} \mathrm{T}_{\mathrm{j}}, \rho=\mathrm{n}_{\mathrm{i}} \mathrm{m}_{\mathrm{i}}$ is the mass density, $v=\mathrm{n}_{0}\langle\sigma \mathrm{v}\rangle_{\mathrm{x}}$ and $v_{0}=\mathrm{n}_{\mathrm{i}}\langle\sigma \mathrm{v}\rangle_{\mathrm{x}}$ are the ion-neutral and neutral-ion charge exchange frequencies, and $\mathbf{u}_{0}$ is the neutral velocity. The mass flow velocity is given by the parallel, $\mathbf{E} \times \mathbf{B}$ and diamagnetic flows of the ions

$$
\mathbf{u}=\mathrm{u}_{\|} \mathbf{b}+\frac{\mathrm{c}}{\mathrm{B}} \mathbf{b} \times \nabla \phi+\frac{\mathrm{c}}{\mathrm{Zen}_{\mathrm{i}} \mathrm{B}} \mathbf{b} \times \nabla \mathrm{p}_{\mathrm{i}} .
$$

We assume $\mathbf{u}_{0}<<\mathbf{u}$, which allows us to carry out the calculation without introducing an explicit neutral model. An examination of the neutral parallel momentum equation shows that $\mathrm{u}_{\| 0}<<\mathrm{u}_{\|}$is valid in the large neutral mean free path limit, $\lambda_{0} / \mathrm{L}_{\perp}>>$ 1 , where $\lambda_{0}=\mathrm{v}_{0} / v_{0} \approx \mathrm{v}_{\mathrm{i}} / v_{0}$ is the neutral mean free path for charge exchange and $\mathrm{L}_{\perp}$ is the plasma radial scale length. In this limit, the neutral viscosity is balanced by the ion friction term so that $\mathrm{u}_{\| 0} \sim\left(\mathrm{L}_{\perp} / \lambda_{0}\right)^{2} \mathrm{u}_{\|}$.

The plasma viscosity term was omitted from Eq. (3) because we restrict the discussion in this paper to the case where the ion-neutral friction is larger than the parallel ion viscosity in the parallel momentum balance. The neglect of parallel viscosity requires $v \gg \gamma_{\|}$, where $\gamma_{\|}$is the viscous damping rate. Simple estimates give $\gamma_{\|} \approx v_{\mathrm{i}} / \mathrm{L}_{\|}$in the plateau regime and $\gamma_{\|} \approx v_{\mathrm{i}}^{2} /\left(v_{\mathrm{ii}} \mathrm{L} \|^{2}\right)$ in the Pfirsch-Schluter regime. In terms of scale lengths, the plasma viscosity can be neglected when $\lambda / L_{\|}<<1$ in the plateau regime and when $\lambda \lambda_{\mathrm{ii}} / \mathrm{L}_{\|}^{2} \ll 1$ in the Pfirsch-Schluter regime, where $\lambda=\mathrm{v}_{\mathrm{i}} / v$ is the ion mean free path for charge exchange and $\lambda_{\mathrm{ii}}$ is the ion mean free path for ion-ion Coulomb collisions. For typical parameters, $\mathrm{n}_{0} \ll \mathrm{n}_{\mathrm{i}}$ and $\mathrm{L}_{\perp} \ll \mathrm{L}_{\|}$so that the validity conditions for neglect of $\mathbf{u}_{0}$ and neglect of parallel viscosity can be simultaneously satisfied.

We employ the usual toroidal flux coordinates $(\psi, \theta, \varphi)$ defined by

$$
\mathbf{B}=\mathrm{B}_{\zeta} \hat{\mathbf{e}}_{\zeta}+\mathrm{B}_{\theta} \hat{\mathbf{e}}_{\theta}=\mathrm{B}_{\zeta} \hat{\mathbf{e}}_{\zeta}+\frac{1}{\mathrm{R}} \hat{\mathbf{e}}_{\zeta} \times \nabla \psi
$$

where $\psi=$ const. denotes surfaces of constant poloidal magnetic flux. For axisymmetric plasmas $(\partial / \partial \varphi=0)$ we have the following useful relations for any scalar $\mathbf{Q}$ and vector $\mathbf{A}$ : 


$$
\begin{gathered}
\nabla \mathrm{Q}=\hat{\mathbf{e}}_{\psi} \mathrm{RB}_{\theta} \frac{\partial \mathrm{Q}}{\partial \psi}+\hat{\mathbf{e}}_{\theta} \frac{1}{\mathrm{JB}_{\theta}} \frac{\partial \mathrm{Q}}{\partial \theta}, \\
\nabla_{\|} \mathrm{Q}=\frac{1}{\mathrm{JB}} \frac{\partial \mathrm{Q}}{\partial \theta}, \\
\nabla \cdot \mathbf{A}=\frac{1}{\mathrm{~J}} \frac{\partial}{\partial \psi}\left(\mathrm{JRB}_{\theta} \mathrm{A}_{\psi}\right)+\frac{1}{\mathrm{~J}} \frac{\partial}{\partial \theta}\left(\frac{\mathrm{A}_{\theta}}{\mathrm{B}_{\theta}}\right), \\
\langle\nabla \cdot \mathbf{A}\rangle=\frac{1}{v} \frac{\partial}{\partial \psi} \int \mathrm{d} \theta \mathrm{JRB}_{\theta} \mathrm{A}_{\psi}=\frac{1}{v} \frac{\partial}{\partial \psi} v\left\langle\mathrm{RB}_{\theta} \mathrm{A}_{\psi}\right\rangle,
\end{gathered}
$$

where $A_{i}=\hat{\mathbf{e}}_{i} \cdot \mathbf{A}, \quad J$ is the Jacobian, $v=\int d \theta J=\int d s / B$ with $s$ the distance along the field line, and the flux surface average $\langle\mathrm{Q}>$ is defined as

$$
\langle\mathrm{Q}\rangle=\frac{1}{v} \int \mathrm{d} \theta \mathrm{JQ}
$$

and has the property that it annihilates the operator $\mathrm{B} \nabla_{\|}$.

The plasma ambipolarity constraint relates the flux surface average of the external sources $\left(\nabla \cdot \mathbf{J}_{\mathrm{S}}\right)$ to that of the plasma response. The simplest derivation involves solving for $\mathrm{J}_{\mathrm{p} \psi}$ from the toroidal component of momentum balance, Eq. (3), and relating it to $\mathrm{J}_{\mathrm{s} \psi}$ by means of Eq. (2). Taking the flux surface average of this expression, we obtain the following expression:

$$
\begin{gathered}
-\frac{1}{\mathrm{c}}\left\langle\mathrm{RB}_{\theta} \mathrm{J}_{\mathrm{s} \psi}\right\rangle=\frac{1}{\mathrm{c}}\left\langle\mathrm{RB}_{\theta} \mathrm{J}_{\mathrm{p} \psi}\right\rangle=\left\langle\nabla \cdot\left(\rho \mathrm{uu}_{\zeta} \mathrm{R}\right)+\rho v \mathrm{u}_{\zeta} \mathrm{R}\right\rangle, \\
=\frac{1}{v} \frac{\partial}{\partial \psi} v\left\langle\mathrm{R}^{2} \mathrm{~B}_{\theta} \rho \mathrm{u}_{\psi} \mathrm{u}_{\zeta}\right\rangle+\left\langle\rho \mathrm{u}_{\zeta} \mathrm{R}\right\rangle .
\end{gathered}
$$

In writing Eq. (11), we have used Eq. (9) and the vector identity

$$
\mathrm{R} \hat{\mathbf{e}}_{\psi} \cdot \nabla \cdot(\mathbf{T})=\nabla \cdot\left(\mathbf{T} \cdot \mathbf{e}_{\psi} \mathrm{R}\right),
$$

which is valid for any symmetric tensor $\mathbf{T}$.

It can be shown that Eqs. (2) and (3) imply "automatic ambipolarity" $\left(\mathrm{J}_{\mathrm{p} \psi}=0\right)$ in the simple case with no source terms $\left(\nabla \cdot \mathbf{J}_{\mathrm{S}}=0\right)$, no parallel flows $\left(\mathrm{u}_{\|}=0\right)$, and no neutral friction $(v=0)$. In this limit, it follows from the parallel component of Eq. (3) that $\nabla_{\|} p=$ 
0 , so that $\mathrm{p}=\mathrm{p}(\psi)$ and $\mathrm{J}_{\mathrm{p} \psi}=0$. It can be shown that automatic ambipolarity also follows from Eq. (11), because the term involving departures from flux surfaces $\left(\mathrm{u}_{\perp \psi}\right)$ vanishes when $v=0$. This result follows from the solution of the equations described in Sec. IV. Thus, the right-hand-side (rhs) of Eq. (11) is proportional to the perpendicular plasma conductivity arising from ion-neutral collisions (charge exchange).

It will be seen subsequently [from estimates based on Eqs. (16) and (17)] that the term involving $\mathrm{u}_{\psi}$ is negligible compared to the second term on the rhs of Eq. (11) and can be dropped when the poloidal gyroradius is small, i.e. $\rho_{\theta} / L_{\perp}=\left(\rho_{s} / L_{\perp}\right)\left(B / B_{\theta}\right)<<1$ where $\rho_{\mathrm{s}}=\mathrm{c}_{\mathrm{S}} / \Omega_{\mathrm{i}}$ and $\mathrm{L}_{\perp}$ is the radial scale length. This ordering will be assumed here.

The ambipolarity constraint, Eq. (11), can be put in another useful form. The source radial current density $\mathrm{J}_{\mathrm{S} \psi}$ is related to the total radial current flowing through each flux surface,

$$
\mathrm{I}_{\mathrm{S}}(\psi)=\oint \mathrm{d} \mathbf{A} \cdot \mathbf{J}_{\mathrm{s}}=\oint \mathrm{dV} \nabla \cdot \mathbf{J}_{\mathrm{S}}=2 \pi \int_{0}^{\psi} \mathrm{d} \psi^{\prime} v\left\langle\nabla \cdot \mathbf{J}_{\mathrm{s}}\right\rangle
$$

Making use of Eq. (9) we obtain

$$
\left\langle\mathrm{RB}_{\theta} \mathrm{J}_{\mathrm{s} \psi}\right\rangle=\frac{\mathrm{I}_{\mathrm{s}}(\psi)}{2 \pi v}
$$

and using the toroidal component of Eq. (4) to obtain $\mathrm{u}_{\zeta}$, Eq. (11) becomes

$$
\frac{\mathrm{I}_{\mathrm{s}}(\psi)}{2 \pi v}=\left\langle\frac{\rho v \mathrm{c}^{2}}{\mathrm{~B}^{2}} \mathrm{R}^{2} \mathrm{~B}_{\theta}^{2}\left(\frac{\partial \phi}{\partial \psi}+\frac{1}{\mathrm{en}} \frac{\partial \mathrm{p}_{\mathrm{i}}}{\partial \psi}\right)-\frac{\mathrm{cRB} \zeta}{\mathrm{B}} \rho v \mathrm{u}_{\|}\right\rangle
$$

where $\mathrm{n} \equiv \mathrm{n}_{\mathrm{e}}=\mathrm{Zn}_{\mathrm{i}}$ by quasineutrality.

A complete solution of the ambipolarity problem requires that Eq. (14) be supplemented by two other independent constraints. Here, we use Eq. (1) and the parallel pressure balance constraint, obtained by taking the component of Eq. (3) along B:

$$
\begin{gathered}
\mathrm{B} \nabla_{\|}\left(\frac{\rho \mathrm{u}_{\|}}{\mathrm{B}}\right)+\mathrm{B} \nabla_{\|}\left(\frac{\rho \varpi}{\mathrm{B}^{2}}\right)+\frac{1}{\mathrm{~J}} \frac{\partial}{\partial \psi}\left(\mathrm{JRB}_{\theta} \rho \mathrm{u}_{\psi}\right)=0, \\
\nabla_{\|} \mathrm{p}+\frac{\rho \overline{\mathrm{B}}}{\mathrm{B}}\left(\nabla_{\|} \mathrm{u}_{\|}-\mathbf{u} \cdot \mathbf{\kappa}\right)+\rho \mathrm{vu}_{\|}=0,
\end{gathered}
$$


where

$$
\begin{gathered}
\frac{\varpi}{\mathrm{B}} \equiv \frac{\mathrm{B}}{\mathrm{B}_{\theta}} \widehat{\mathbf{e}}_{\zeta} \times \widehat{\mathbf{e}}_{\psi} \cdot \mathbf{u}_{\perp}=\frac{\mathrm{Bu}_{\theta}}{\mathrm{B}_{\theta}}=\mathrm{RB} \zeta \frac{\mathrm{c}}{\mathrm{B}}\left(\frac{\partial \phi}{\partial \psi}+\frac{1}{\mathrm{en}} \frac{\partial \mathrm{p}_{\mathrm{i}}}{\partial \psi}\right), \\
\mathrm{u}_{\psi} \equiv-\frac{\mathrm{cB} \zeta}{\mathrm{BB}_{\theta}}\left(\nabla_{\|} \phi+\frac{1}{\text { en }} \nabla_{\|} \mathrm{p}_{\mathrm{i}}\right) .
\end{gathered}
$$

In Eqs. (14) - (18) some small terms have been dropped, and it has been checked $a$ posteriori that this set of equations is self-consistent in the ordering discussed subsequently. With this caveat, we point out that these nonlinear equations are valid for a wide class of axisymmetric toroidal configurations. The analytic solution of these equations requires some approximations, which will be discussed in the next section.

\section{The Physical Model}

\section{A. Approximations and Ordering}

To obtain an explicit relation between $\mathrm{I}_{\mathrm{S}}$ and $\mathrm{E}_{\mathrm{r}}$, we solve for the variation in the flux surface of the density, pressure, potential, and parallel velocity. A consistent ordering is needed that permits linearization of Eqs. (15) and (16), with the poloidallyvarying pieces treated as perturbations of the one-dimensional cylindrical equilibrium due to the effects of toroidicity. Thus, we assume that the poloidal variation of $\rho, p$ and $\phi$ is driven by the variation of the geodesic curvature, and is of the same order as that of the magnetic field $\left(\sim \mathrm{a} / \mathrm{L}_{\|}\right)$. In this ordering the perpendicular drift and parallel flow velocities are small compared to the sound speed. The quadratic nonlinear beating of the plasma perturbations with the magnetic field perturbation gives lowest order contributions to the flux surface average in Eq. (14), and evaluating these terms gives the desired voltagecurrent relation.

The first step is to express each physical quantity as the sum of surface-averaged and poloidally-varying contributions,

$$
\mathrm{Q}=\overline{\mathrm{Q}}+\widetilde{\mathrm{Q}}
$$

where $\overline{\mathrm{Q}}=\langle\mathrm{Q}\rangle, \widetilde{\mathrm{Q}}=\mathrm{Q}-\langle\mathrm{Q}\rangle$, and $\widetilde{\mathrm{Q}}<\overline{\mathrm{Q}}$ is required in the following treatment. Several assumptions are then made to simplify the calculation of the perturbations. First, we assume that $\mathrm{T}_{\mathrm{i}} \sim \mathrm{T}_{\mathrm{e}}=\mathrm{T}$ and that the parallel thermal conductivity is large, so that the 
temperatures are constant on a flux surface. Then the pressure and density variations are related by

$$
\tilde{\mathrm{p}}=\mathrm{c}_{\mathrm{s}}^{2}\left(1+\frac{\mathrm{T}_{\mathrm{i}}}{\mathrm{T}_{\mathrm{e}}}\right) \tilde{\rho}
$$

where $\mathrm{c}_{\mathrm{s}}=\left(\mathrm{T} / \mathrm{m}_{\mathrm{i}}\right)^{1 / 2}$. Using the isothermal approximation in Ohm's Law yields an expression for the poloidally-varying potential:

$$
\nabla_{\|} \tilde{\phi}=\frac{\mathrm{T}_{\mathrm{e}}}{\mathrm{e} \rho} \nabla_{\|} \tilde{\rho}
$$

Finally, we neglect the poloidal variation of the neutral density, and hence of the ionneutral collision frequency $(\widetilde{v} \rightarrow 0)$, which is valid when $\widetilde{v} \bar{u}_{\|}<<\bar{v} \tilde{u}_{\|}$. This approximation is consistent with the ordering used in our calculation, as we now show.

We adopt an ordering in which the small parameters are $\varepsilon=a / L_{\|}$and $\rho_{\theta} / L_{\perp}$, where $\rho_{\theta}$ is the poloidal gyroradius. Another fundamental dimensionless parameter in the present theory is $\alpha \approx v \omega_{\mathrm{E}} / \omega_{\|}^{2} \approx(\mathrm{e} \bar{\phi} / \mathrm{T})\left(\rho_{\theta} / \mathrm{L}_{\perp}\right)\left(\mathrm{L}_{\|} / \lambda\right)$, which is assumed to be of order unity in order to treat the new bifurcation physics associated with charge exchange. Analysis of Eqs. (14)-(17), (20) and (21) then gives the size of the important physical quantities:

$$
\begin{aligned}
& \frac{\mathrm{e} \tilde{\phi}}{\mathrm{T}} \sim \frac{\tilde{\rho}}{\rho} \sim \frac{\tilde{\mathrm{p}}}{\mathrm{p}} \sim \varepsilon, \quad \frac{\tilde{\mathrm{u}}_{\psi}}{\mathrm{c}_{\mathrm{s}}} \sim \varepsilon \frac{\rho_{\theta}}{\mathrm{L}_{\perp}} \frac{\mathrm{L}_{\perp}}{\mathrm{L}_{\|}}, \\
& \frac{\overline{\mathrm{u}}_{\perp \theta}}{\mathrm{c}_{\mathrm{s}}} \sim \frac{\tilde{\mathrm{u}}_{\|}}{\mathrm{c}_{\mathrm{s}}} \sim \varepsilon \delta, \quad \frac{\tilde{\mathrm{u}}_{\perp \theta}}{\mathrm{c}_{\mathrm{s}}} \sim \varepsilon^{2} \frac{\rho_{\theta}}{\mathrm{L}_{\perp}}, \quad \frac{\overline{\mathrm{u}}_{\|}}{\mathrm{c}_{\mathrm{s}}}<\varepsilon^{2} \delta,
\end{aligned}
$$

where $\delta=\lambda / L_{\|}$is not necessarily small. The ordering in Eq. (22) implies the existence of well-posed flux surfaces for $\varepsilon<<1$ and the importance of the perturbed parallel flow ( $\tilde{\mathrm{u}}_{\|}$ $\sim \overline{\mathrm{u}}_{\theta}$ ). The last estimate is based on the flux-surface average of B times Eq. (16) noting that $\left\langle\mathrm{B} \nabla_{\|} \mathrm{p}\right\rangle=0$, and implies that $\overline{\mathrm{u}}_{\|}<<\widetilde{\mathrm{u}}_{\|}$, which differs from the usual ordering in neoclassical theory. Thus, $\overline{\mathrm{u}}_{\|}$does not enter the linearized equations. This ordering has the important property that it allows the "quasilinear" (i.e. the quadratic perturbation beat) terms in Eq. (14) to compete.

Our assumption that $\alpha \sim 1$ implies orderings for $\delta$ and $\mathrm{e} \bar{\phi} / \mathrm{T}$ which were used in deriving Eq. (22). The general ordering encompasses two sub-cases. In the first case, we 
assume e $\bar{\phi} / \mathrm{T} \sim 1$ so that $\alpha \sim 1$ implies $\delta \sim \rho_{\theta} / \mathrm{L}_{\perp} \ll<1$; in the second case we assume e $\bar{\phi}$ $/ \mathrm{T} \gg>1$, implying that $\delta$ can be of order unity. In the latter case, the poloidal Mach number $\mathrm{M}_{\mathrm{p}}=\left(\mathrm{u}_{\theta} / \mathrm{c}_{\mathrm{s}}\right)\left(\mathrm{B}_{\zeta} / \mathrm{B}_{\theta}\right)$ can be of order unity. For the first case, the neglect of plasma viscosity is valid in both the plateau and Pfirsch-Schlüter regimes; in the second case, the neglect of viscosity can be justified in the Pfirsch-Schlüter regime when $\lambda_{\mathrm{ii}}$ is sufficiently short. We will show that the ambipolarity condition takes slightly different forms for the two cases.

\section{B. Reduced Equations}

We now apply the approximations described in the preceding section to reduce the ambipolarity problem to its simplest form. Treating the poloidally-varying pieces as perturbations, we linearize Eqs. (15) and (16) to obtain

$$
\begin{gathered}
\rho \nabla_{\|} \tilde{\mathrm{u}}_{\|}-\frac{2 \rho \varpi \mathrm{B}_{\theta}}{\mathrm{B}_{\zeta}^{2}} \widetilde{\kappa}_{\theta}+\left(\frac{\varpi}{\mathrm{B}}-\delta_{2} \frac{\Phi_{0}}{\mathrm{~B}}\right) \nabla_{\|} \tilde{\rho}=0, \\
\nabla_{\|} \tilde{\mathrm{p}}+\delta_{1} \frac{\rho \Phi}{\mathrm{B}} \nabla_{\|} \tilde{\mathrm{u}}_{\|}+\rho v \widetilde{\mathrm{u}}_{\|}=\delta_{1} \frac{\rho \Phi^{2} \mathrm{~B}_{\theta}}{\mathrm{BB}_{\zeta}^{2}} \widetilde{\kappa}_{\theta},
\end{gathered}
$$

where

$$
\frac{\varpi_{0}}{\mathrm{~B}} \equiv \mathrm{RB}_{\zeta} \frac{\mathrm{c}}{\mathrm{B}} \frac{1}{\mathrm{ne}}\left(\frac{\partial \mathrm{p}_{\mathrm{e}}}{\partial \psi}+\frac{\partial \mathrm{p}_{\mathrm{i}}}{\partial \psi}\right)
$$

Here and in what follows, we keep the tildes to denote poloidally-varying quantities (including the geodesic curvature) but omit the overbars on averaged quantities to simplify the notation. We introduce the parameters $\delta_{1}$ and $\delta_{2}$ to permit a unified treatment of both sub-cases described in the previous section: the case with $\mathrm{e} \bar{\phi} / \mathrm{T} \sim 1$ corresponds to $\delta_{1}=0, \delta_{2}=1$ and the case with $\mathrm{e} \bar{\phi} / \mathrm{T}>1$ corresponds to $\delta_{1}=1, \delta_{2}=0$. The poloidal variation of the magnetic field is described in Eq. (23) by $\widetilde{\kappa}_{\theta}$, which is defined by

$$
\widetilde{\boldsymbol{\kappa}}_{\theta} \equiv \hat{\mathbf{e}}_{\theta} \cdot \boldsymbol{\kappa}=\frac{\mathrm{B}_{\zeta}^{2}}{\mathrm{~B}^{2} \mathrm{~B}_{\theta}} \nabla_{\|} \mathrm{B}=\frac{\mathrm{B}_{\zeta}^{2}}{\mathrm{JB}^{3} \mathrm{~B}_{\theta}} \frac{\partial \mathrm{B}}{\partial \theta}
$$


and is related to the true geodesic curvature $\tilde{\kappa}_{\mathrm{g}} \equiv \mathbf{b} \times \mathbf{e}_{\psi} \cdot \kappa$ by $\widetilde{\kappa}_{\theta}=(\mathrm{B} \zeta / \mathrm{B}) \widetilde{\kappa}_{\mathrm{g}}$.

Using Eqs. (20) and (21), we can recast Eqs. (23) and (24) in terms of two variables (e.g. $\tilde{\mathrm{p}}$ and $\tilde{\mathrm{u}} \|$ ) with a drive term proportional to $\tilde{\kappa}_{\theta}$. These equations imply that the poloidal variation of the geodesic curvature produces a corresponding poloidal variation in the other plasma quantities. This is the manifestation in the present model of the Pfirsch-Schlüter flows of neoclassical theory.

Next we substitute the linearized quantities into Eq. (14) and keep only the lowest-order quadratic terms to obtain the desired ambipolarity constraint. The $\left\langle\rho v u_{\|} / \mathrm{B}\right\rangle$ term in Eq. (14) is treated as follows. We eliminate $\rho v u_{\|}$using the nonlinear parallel pressure balance equation, Eq. (16). Each of the terms is then manipulated to extract an explicit curvature. The terms with $\nabla_{\|} \mathrm{p}$ or $\nabla_{\|} \mathrm{u}_{\|}$are integrated by parts to obtain $\nabla_{\|} \mathrm{B} \propto \tilde{\kappa}_{\theta}$ [see Eq. (26)]. Finally, a term proportional to $\left\langle\tilde{\rho} v \tilde{u}_{\|}\right\rangle$is manipulated using the following identity, obtained by multiplying the linearized continuity equation by $\tilde{\rho}$ and averaging,

$$
\left\langle\tilde{\rho} \nabla_{\|} \tilde{\mathrm{u}}_{\|}\right\rangle=\left\langle\frac{2 \sigma \mathrm{B}_{\theta}}{\mathrm{B}_{\zeta}^{2}} \tilde{\rho} \tilde{\kappa}_{\theta}\right\rangle .
$$

It is also useful to note that the terms containing $\Phi$ also have the factor $\delta_{1}$ and hence only matter in case 2 , where $\mathrm{e} \bar{\phi} / \mathrm{T}>>1$. In this limit it can be shown that the perturbation of

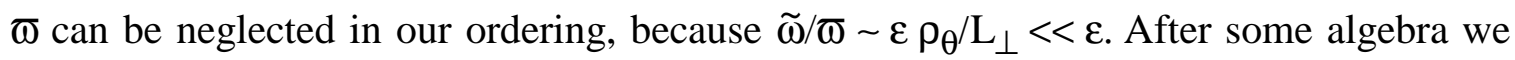
obtain

$$
\frac{\mathrm{I}_{\mathrm{s}}(\psi)}{2 \pi v}=\left\langle\frac{\mathrm{cRB} \mathrm{B}_{\theta}}{\mathrm{B}}\left[\frac{\rho \Phi}{\mathrm{B}}\left(\frac{v \mathrm{~B}_{\theta}}{\mathrm{B}_{\zeta}}+\delta_{1} \frac{3 \mathrm{~B}}{\mathrm{~B}_{\zeta}} \tilde{\mathrm{u}}_{\|} \tilde{\kappa}_{\theta}\right)+\frac{2 \mathrm{~B}}{\mathrm{~B}_{\zeta}} \tilde{\mathrm{p}}_{\tilde{\kappa}_{\theta}}+\delta_{1} \frac{\varpi^{2}}{\mathrm{BB}_{\zeta}} \tilde{\rho} \tilde{\kappa}_{\theta}\right]\right\rangle .
$$

The first term $(\propto \rho \vee \sigma)$ gives the well-known linear relation between $I_{s}$ and $E_{\psi}$ and involves only surface-averaged quantities; the contributions to this term from the perturbations are higher order in $\varepsilon$. The remaining terms involve products of the perturbed quantities and are of the same order as the first term.

One could carry out the solution of Eqs. (23) and (24) and the integral in Eq. (27) numerically for general axisymmetric toroidal geometry, even including X-points. However, for the purpose of illustrating the basic physics, it is more convenient to pursue 
an analytic solution in the cylindrical tokamak limit, as described in the following section.

\section{Analytic Solution}

\section{A. Poloidal Variation}

After some straightforward algebra, the linearized equations (23) and (24) can be rewritten in terms of the variables $X=\tilde{p}$ and $Y=\rho v L_{\|} \widetilde{\mathrm{u}}_{\|}$as follows:

$$
\begin{gathered}
\mathrm{L}_{\|} \nabla_{\|} \mathrm{Y}+\alpha \mathrm{L}_{\|} \nabla_{\|} \mathrm{X}=-\beta \mathrm{R} \tilde{\kappa}_{\theta} \\
\mathrm{L}_{\|} \nabla_{\|} \mathrm{X}+\left(\delta_{1} \mu \mathrm{L}_{\|} \nabla_{\|}+1\right) \mathrm{Y}=-\delta_{1} \frac{\mu \beta \mathrm{R}}{2} \widetilde{\kappa}_{\theta},
\end{gathered}
$$

where the coefficients are defined by

$$
\begin{aligned}
& \alpha=\left(\frac{\mathrm{L}_{\|}}{\lambda}\right)^{2} \frac{\mathrm{T}_{\mathrm{i}}}{\mathrm{T}_{\mathrm{e}}+\mathrm{T}_{\mathrm{i}}} \mu^{*}, \quad \beta=-\frac{2 \mathrm{p}_{\mathrm{i}} \mathrm{L}_{\|} \mathrm{BB}_{\theta}}{\mathrm{RB}_{\zeta}{ }^{2}}\left(\frac{\mathrm{L}_{\|}}{\lambda}\right)^{2} \mu, \\
& \mu \equiv \frac{\varpi}{v L_{\|} \mathrm{B}}=\frac{-\mathrm{cB} \zeta}{v \mathrm{~L}_{\|} \mathrm{BB}_{\theta}}\left(\mathrm{E}_{\psi}-\frac{\mathrm{RB}_{\theta}}{\mathrm{en}} \frac{\partial \mathrm{p}_{\mathrm{i}}}{\partial \psi}\right) \\
& =\frac{\mathrm{qR}}{\mathrm{L}_{\|}} \frac{\mathrm{B}}{\mathrm{B}_{\zeta}}\left(\frac{\omega_{\mathrm{E}}+\omega_{*_{\mathrm{i}}}}{v}\right) \text {, } \\
& \mu^{*} \equiv \frac{1}{v L_{\|}}\left(\frac{\varpi}{B}-\delta_{2} \frac{\varpi_{0}}{\mathrm{~B}}\right)=\mu-\delta_{2} \frac{\mathrm{cB}_{\zeta}}{\mathrm{vL}_{\|} \mathrm{BB}_{\theta}} \frac{\mathrm{RB}_{\theta}}{\mathrm{en}}\left(\frac{\partial \mathrm{p}_{\mathrm{e}}}{\partial \psi}+\frac{\partial \mathrm{p}_{\mathrm{i}}}{\partial \psi}\right) \\
& =\mu-\delta_{2} \frac{\mathrm{qR}}{\mathrm{L}_{\|}} \frac{\mathrm{B}}{\mathrm{B}_{\zeta}}\left(\frac{\omega_{*_{\mathrm{i}}}-\omega_{*_{\mathrm{e}}}}{\mathrm{v}}\right) .
\end{aligned}
$$

Here, $\lambda=\mathrm{v}_{\mathrm{i}} / \mathrm{v}, \mathrm{q}=\mathrm{aB} \zeta /\left(\mathrm{RB}_{\theta}\right)$ is the cylindrical safety factor, $\mathrm{E}_{\psi}=-\mathrm{RB}_{\theta}(\partial \phi \partial \psi), \omega_{\mathrm{E}}=$ $\mathrm{v}_{\mathrm{E}} / \mathrm{a}$ is the poloidal $\mathbf{E} \times \mathbf{B}$ drift frequency, and $\omega_{* \mathrm{j}}=\mathrm{v}_{* \mathrm{pj}} / \mathrm{a}$ are the diamagnetic drift frequencies $\left(\mathrm{j}=\mathrm{i}\right.$, e) with $\mathrm{V}_{* \mathrm{pj}}=\sigma_{\mathrm{j}} \mathrm{cB} /(\mathrm{en}) \nabla_{\perp} \mathrm{p}_{\mathrm{j}}$. The drift frequencies are given in flux coordinates by

$$
\omega_{E}=-\frac{\mathrm{cE}_{\psi} \mathrm{B}_{\zeta}}{\mathrm{aB}^{2}}, \quad \omega_{* j}=\sigma_{j} \frac{\mathrm{cRB}_{\theta} \mathrm{B}_{\zeta}}{\mathrm{aB}^{2} \text { ne }} \frac{\partial \mathrm{p}_{j}}{\partial \psi}
$$


where $\sigma_{\mathrm{i}}=+1$ and $\sigma_{\mathrm{e}}=-1$. The coefficients $\alpha, \beta, \mu$ and $\mu *$ involve the perpendicular $\mathbf{E} \times \mathbf{B}$ and diamagnetic drifts; the terms in Eqs. (28) and (29) without these coefficients arise from the parallel flow and the curvature-induced poloidal variations.

In general geometry, the factors of $\mathrm{R}, \mathrm{B}_{\zeta}, \mathrm{B}_{\theta}$ in these equations, and thus the coefficients $\alpha, \beta$ and $\mu$, are not constant along the field line. As we are seeking an analytic solution, we now take the cylindrical tokamak limit $(\mathrm{R} \rightarrow \infty)$ in which all of these quantities are constant to lowest order in $\varepsilon=\mathrm{a} / \mathrm{L}_{\|}$. In this limit, to lowest order we have that $\mathrm{L}_{\|}=\mathrm{qR}, \mathrm{B}_{\zeta} / \mathrm{B}=1, \mathrm{~B}_{\theta} / \mathrm{B}_{\zeta}=\mathrm{a} / \mathrm{L}_{\|}, \mathrm{L}_{\|} \nabla_{\|}=\mathrm{d} / \mathrm{d} \theta$, and the geodesic curvature is given by

$$
\tilde{\mathrm{\kappa}}_{\theta}=\frac{1}{\mathrm{R}} \sin \theta,
$$

where we have chosen $\theta=0$ at the outer midplane. Defining $\hat{\mu}=\delta_{1} \mu$, we obtain the following solution for the amplitudes

$$
\begin{aligned}
& X=\frac{\beta}{\alpha^{2}+(1-\hat{\mu} \alpha)^{2}}\left\{\left[\alpha\left(1+\hat{\mu}^{2} / 2\right)-\hat{\mu} / 2\right] \cos \theta-(1-\hat{\mu} \alpha / 2) \sin \theta\right\}, \\
& Y=\frac{\beta}{\alpha^{2}+(1-\hat{\mu} \alpha)^{2}}(1-\hat{\mu} \alpha / 2)\{(1-\hat{\mu} \alpha) \cos \theta+\alpha \sin \theta\} .
\end{aligned}
$$

Thus, both the pressure $(\mathrm{X})$ and the parallel flow $(\mathrm{Y})$ have components in-phase and outof-phase with the poloidal variation of the geodesic curvature.

\section{B. Ambipolarity Constraint}

The final form of the ambipolarity constraint in the cylindrical tokamak limit is obtained by combining Eqs. (20), (21), (27), (34) and (35) and carrying out the flux surface average by integrating over $\theta$. The result can be put in the form of a relation between the normalized radial source current and the radial electric field:

$$
\begin{aligned}
\frac{\mathrm{I}_{\mathrm{S}}(\psi)}{4 \pi^{2}{ }_{\mathrm{aR}}} & \equiv-\sigma_{\perp}\left(\mathrm{E}_{\psi}-\frac{\mathrm{RB}_{\theta}}{\mathrm{en}} \frac{\partial \mathrm{p}_{\mathrm{i}}}{\partial \psi}\right), \\
& =-\sigma_{\perp 0}\left(\mathrm{E}_{\psi}-\frac{\mathrm{RB}_{\theta}}{\mathrm{en}} \frac{\partial \mathrm{p}_{\mathrm{i}}}{\partial \psi}\right)\left[1+\frac{2 \mathrm{q}^{2}(1-\hat{\mu} \alpha)(1-\hat{\mu} \alpha / 2)}{\alpha^{2}+(1-\hat{\mu} \alpha)^{2}}\right],
\end{aligned}
$$


where $\hat{\mu}=\delta_{1} \mu$. In writing Eq. (36), we have used the cylindrical result that $v \equiv \int \mathrm{d} / \mathrm{B}=$ $2 \pi \mathrm{a} / \mathrm{B}_{\theta}$ and for comparison with earlier work we have introduced a reference conductivity $\sigma_{\perp 0} \equiv\left(\rho v c^{2} / \mathrm{B}^{2}\right)$ such that

$$
\sigma_{\perp 0}\left(\mathrm{E}_{\psi}-\frac{\mathrm{RB}_{\theta}}{\text { en }} \frac{\partial \mathrm{p}_{\mathrm{i}}}{\partial \psi}\right)=\frac{\mathrm{cRB}_{\zeta}}{2 \mathrm{~B}^{2} \mathrm{~L}_{\|}^{2}} \beta \equiv-\frac{\mathrm{acp}}{\mathrm{BL}_{\|}^{2}} \xi
$$

At this point, it is useful to replace the coefficients $\{\alpha, \beta\}$ of the linearized equations by new parameters $\left\{\xi, \xi_{d}\right\}$, which characterize the radial electric field and the diamagnetic drifts. The parameter $\xi$ was defined in Eq. (37), and $\xi_{\mathrm{d}}$ is defined as

$$
\xi_{\mathrm{d}} \equiv\left(\frac{\mathrm{L}_{\|}}{\lambda}\right)^{2}\left(\frac{\mathrm{T}_{\mathrm{i}}}{\mathrm{T}_{\mathrm{e}}+\mathrm{T}_{\mathrm{i}}}\right)\left(\frac{\omega_{*_{\mathrm{e}}}-\omega_{*_{\mathrm{i}}}}{v}\right)
$$

so that

$$
\xi=\alpha-\delta_{2} \xi_{d}=\left(\frac{L_{\|}}{\lambda}\right)^{2}\left(\frac{T_{i}}{T_{e}+T_{i}}\right)\left(\frac{\omega_{E}+\omega_{*_{i}}}{v}\right) .
$$

Thus, the plasma conductivity $\sigma_{\perp}$ in the presence of ion-neutral friction depends on four independent parameters: $\mathrm{q}, \xi, \xi_{\mathrm{d}}$ and $\delta \equiv \lambda / \mathrm{L}_{\|}$. To lowest order in $\varepsilon$, the latter three parameters can be rewritten in terms of characteristic frequencies as

$$
\xi=\left(\frac{\mathrm{T}_{\mathrm{e}}}{\mathrm{T}_{\mathrm{e}}+\mathrm{T}_{\mathrm{i}}}\right) \frac{v\left(\omega_{\mathrm{E}}+\omega_{*_{\mathrm{i}}}\right)}{\omega_{\|}^{2}}, \quad \xi_{\mathrm{d}}=\left(\frac{\mathrm{T}_{\mathrm{e}}}{\mathrm{T}_{\mathrm{e}}+\mathrm{T}_{\mathrm{i}}}\right) \frac{v \omega_{*} \mathrm{p}}{\omega_{\|}^{2}}, \quad \delta=\left(\frac{\mathrm{T}_{\mathrm{i}}}{\mathrm{T}_{\mathrm{e}}}\right)^{1 / 2} \frac{\omega_{\|}}{v},
$$

where $\omega_{\|}=c_{S} / L_{\|}$is the parallel transit frequency, $\omega_{E}$ is the poloidal $\mathbf{E} \times \mathbf{B}$ drift frequency, and $\omega_{* \mathrm{p}}=\left(\omega_{*_{\mathrm{e}}}-\omega_{*_{\mathrm{i}}}\right)$ is the diamagnetic drift frequency based on the total presssure, $\mathrm{p}=$ $\mathrm{p}_{\mathrm{e}}+\mathrm{p}_{\mathrm{i}}$, and the perpendicular drift frequencies are defined in Eq. (33). Both $\xi$ and $\xi_{\mathrm{d}}$ are assumed to be comparable in size to $\alpha$, which was taken to be order unity in carrying out the derivation. Note that the radial electric field appears only in the parameter $\xi$, and one has that $\xi \propto-\mathrm{E}_{\psi}$ when $\mathrm{T}_{\mathrm{i}} \rightarrow 0$. The parameter $\xi_{\mathrm{d}}$ contains the diamagnetic drifts. The parameters $\mathrm{q}$ and $\delta$ both depend on the magnetic geometry, and for fixed geometry $\delta$ characterizes the ion-neutral collisionality.

Taking the limit $\mu \rightarrow 0$ in Eq. (36), one finds that $v \rightarrow \infty$ implies $\left\{\alpha, \xi, \xi_{d}\right\} \rightarrow \infty$, $\delta \rightarrow 0$ and the plasma conductivity goes to the well-known result $\sigma_{\perp} \rightarrow \sigma_{\perp 0}$; in the 
opposite limit of small but finite $v$ such that $\left\{\alpha, \xi, \xi_{d}\right\} \rightarrow 0$, Eq. (36) also yields the standard result 9,11 that $\sigma_{\perp} \rightarrow \sigma_{\perp 0}\left(1+2 q^{2}\right)$. For intermediate ion-neutral collisionality and large drift frequencies, our ambipolarity constraint (36) contains additional physics which leads to a new result, viz. the possibility of multiple roots in the ambipolar constraint and bifurcated equilibrium states due to the ion-neutral frictional force resulting from charge exchange.

We illustrate this point by plotting the dimensionless radial current $\mathrm{I}=\left(\mathrm{BL}_{\|}{ }^{2} \mathrm{acp}\right)$ $\left(\mathrm{I}_{\mathrm{S}} / 4 \pi^{2} \mathrm{aR}\right)$ versus the electric field parameter $\xi$, with the other parameters held fixed, for each of the two cases discussed in Sec. III A. To simplify the discussion, we set $\mathrm{T}_{\mathrm{e}}=\mathrm{T}_{\mathrm{i}}$ in the remainder of this section. In the limit of small $\xi$, Eq. (36) can be manipulated to give the standard linear voltage-current relation, $I(\xi)=\xi\left(1+2 q^{2}\right)$, as expected in the absence of viscosity. Non-monotonic behavior is obtained when $\xi$ is allowed to be of order unity, as we now show.

In the first case $\left(\delta_{1}=0, \delta_{2}=1\right)$, Eq. (36) yields the relation

case 1:

$$
\mathrm{I}(\xi)=\xi\left[1+\frac{2 \mathrm{q}^{2}}{1+\left(\xi+\xi_{\mathrm{d}}\right)^{2}}\right],
$$

so that the function $\mathrm{I}(\xi)$ depends on the two parameters $\mathrm{q}$ and $\xi_{\mathrm{d}}$. Recall that this case employs the orderings e $\bar{\phi} / \mathrm{T} \sim 1$ and $\delta<<1$, implying small $\mathbf{E} \times \mathbf{B}$ drifts, strong ion-neutral collisionality and short ion-neutral mean free path. In this limit, the perpendicular drift terms do not enter into lowest-order parallel pressure balance [ Eq. (24)].

A plot of the function $\mathrm{I}(\xi)$ in Eq. (41) is shown in Fig. 1 for fixed $q$ and two values of the diamagnetic parameter $\xi_{\mathrm{d}}$. It is apparent from Fig. 1 that the equation $\mathrm{I}\left(\xi_{)}\right)=$ $\mathrm{I}_{0}$ has either one or three solutions for $\xi$, depending on the value of $\mathrm{I}_{0}$. Only one root is obtained for either very small or very large values of $\left|\mathrm{I}_{0}\right|$, but an intermediate range of $\mathrm{I}_{0}$ $>0$ yields multiple roots, associated with the local extrema of $\mathrm{I}(\xi)$. The local extrema are produced by the $\mathrm{q}^{2}$ term in Eq. (41) and thus grow larger as q increases. For the case $\mathrm{q}=$ $4, \xi_{d}=0$, shown in Fig. 1(a), the ambipolar current-voltage relation is antisymmetric about $\xi=0$. For either sign of the radial current, the theory predicts a bifurcation induced by neutral friction from a smaller to a larger value of $\left|\mathrm{E}_{\psi}\right|$ as the radial source current is increased. The influence of diagmagnetic drifts is shown in Fig. 1(b) where the case $q=$ 4, $\xi_{\mathrm{d}}=1$ is shown. In this case, the function $\mathrm{I}(\xi)$ is highly asymmetrical, having a local 
extremum for $\xi \sim-\xi_{\mathrm{d}}$. For the usual case of $\xi_{\mathrm{d}}>0$ (corresponding to $\partial \rho / \partial \psi<0$ ), the region of multiple roots occurs for $\xi<0$ and $\mathrm{I}<0$. By definition $\xi \propto \omega_{\mathrm{E}}+\omega_{*_{\mathrm{i}}}$, so this condition implies either that $\mathrm{E}_{\psi}$ is small enough to satisfy $\left|\omega_{\mathrm{E}}\right|<\left|\omega_{*_{\mathrm{i}}}\right|$ or that $\mathrm{E}_{\psi}>0$ (outward-pointing radial electric field). Thus, for case 1 with moderate $\mathrm{q}$ and strong diamagnetic drifts the theory predicts a bifurcation induced by neutral friction only if the current is increased in the negative direction. Finally, we note that as $\mathrm{q}$ is increased at fixed $\xi_{d}$ (not shown) a second, weaker bifurcation is obtained for $\xi<0$ and $\mathrm{I}>0$. This is discussed further in connection with Fig. 2.

In Fig. 2 we show the boundaries in $\xi_{\mathrm{d}}-\mathrm{q}$ parameter space corresponding to single and multiple root solutions of the ambipolar current-voltage relation, $\mathrm{I}(\xi)=\mathrm{I}_{0}$. Below the lowest curve, there is only a single root for any value of $\xi$. In the region between the two boundaries, multiple roots can be obtained for certain values of $\mathrm{I}<0$ but there is only a single root for I > 0, as illustrated in Fig. 1(b). Above the higher curve, multiple roots are obtained for both signs of I. In this upper region, the degree of asymmetry between positive and negative currents depends on the diamagnetic parameter. As $\xi_{d} \rightarrow \infty$ at fixed $\mathrm{q}$, the plasma returns to the middle region where the asymmetry between the two directions of currents is large; on the other hand, as $\xi_{d} \rightarrow 0$ one obtains symmetry between the positive and negative currents, as illustrated in Fig. 1(a).

Turning now to the second case $\left(\delta_{1}=1, \delta_{2}=0\right)$, Eq. (36) yields the following ambipolarity relation

case 2:

$$
\mathrm{I}(\xi)=\xi\left[1+\frac{2 \mathrm{q}^{2}\left(1-\xi^{2} \delta^{2}\right)\left(1-2 \xi^{2} \delta^{2}\right)}{\xi^{2}+\left(1-2 \xi^{2} \delta^{2}\right)^{2}}\right]
$$

where $\mathrm{I}(\xi)$ depends on the two parameters $\mathrm{q}$ and $\delta$. Recall that this result corresponds to the orderings $e \bar{\phi} / \mathrm{T} \gg>1$ and $\delta \geq 1$, implying strong $\mathbf{E} \times \mathbf{B}$ drifts, negligible diamagnetic drifts, and weaker ion-neutral collisionality and a longer ion-neutral mean free path than in case 1. In case 2, the perpendicular $\mathbf{E} \times \mathbf{B}$ drift competes with the parallel flows in lowest-order parallel pressure balance [ Eq. (24)].

A plot of the function $\mathrm{I}(\xi)$ in Eq. (42) is shown in Fig. 3 for the parameters $q=4$ and $\delta=1$. Here, the function $\mathrm{I}(\xi)$ has antisymmetric extrema for $\xi \sim \pm \delta$. There are several differences between this case and the previous one. In case 2, multiple roots are obtained 
below a certain threshold in current; the condition for a unique root is that the magnitude of I exceed a critical value of $\left|\mathrm{I}_{0}\right|$. In case 1, multiple roots could not be obtained for small currents. The number of roots can be larger in case 2 (up to five), because $I(\xi)$ is a fifth order polynomial when the $\mathbf{E} \times \mathbf{B}$ drift terms are retained. Another difference is that the neutral-friction induced bifurcation occurs for either sign of radial current and radial electric field, because the symmetry-breaking diamagnetic effects are unimportant in this ordering. Finally, it should be noted that the linear part of the curve is modified by the $\mathbf{E} \times \mathbf{B}$ drifts to have a larger slope here than in the previous case. In the limit $\xi \rightarrow \infty$, $\mathrm{I}(\xi) \rightarrow \xi\left(1+\mathrm{q}^{2}\right)$ in case 2 whereas $\mathrm{I}(\xi) \rightarrow \xi$ for case 1 .

We conclude this section with two additional remarks. First, it should be kept in mind that the radial current I and the parameters $\mathrm{q}, \xi, \xi_{\mathrm{d}}$ and $\delta$ are all functions of $\psi$, so that the ambipolarity constraint must be solved separately on each flux surface to obtain the radial electric field profile. Second, we point out that the non-monotonic behavior of $\mathrm{I}(\xi)$ on a given flux surface is due to terms involving the products of the surface-averaged perpendicular equilibrium drifts and the perturbations in the continuity and parallel pressure balance equations, viz. the terms with coefficients $\alpha$ and $\mu$ in Eqs. (28) and (29). These terms compete with the perturbed parallel flow in reducing the poloidal variation of the density and pressure. This competition is sensitive to the magnitude of the neutral friction and leads to the bifurcation in the ambipolar voltage-current relation.

\section{Summary and Discussion}

In this paper, we have extended the fluid treatment of the perpendicular plasma conductivity to include the effects of arbitrary ion-neutral collision frequency $v$ and large perpendicular drifts. As discussed in Sec. I, the present calculation of the ambipolar constraint omits the effect of parallel viscosity, and its validity therefore requires $v>\gamma_{\|}$, where $\gamma_{\|}$is the viscous damping rate. This condition can be met for many cases of interest in the edge plasma when the neutral density and the parallel connection length are sufficiently large (e.g. near a separatrix in diverted plasmas). The main result of this calculation is that ion-neutral friction can give a non-monotonic relationship between the radial current and the electric field, leading to a bifurcated plasma equilibrium. This bifurcation may influence the properties of the L-H transition. The physical origin of this effect is a subtle interplay between the surface-averaged equilibrium drifts and the small 
poloidal variations in the pressure and parallel flow around the flux surface generated by the geodesic curvature.

The bifurcation physics described in this paper requires that the flux-surfaceaveraged $\mathbf{E} \times \mathbf{B}$ drift be large enough that the parameter $\alpha \approx v \omega_{E} / \omega_{\|}^{2}$ be order unity. The

ordering $\alpha \sim 1$ encompasses two sub-cases. In the first case, $\omega_{\mathrm{E}} / \omega_{\|} \sim \omega_{\|} / v<<1$, which corresponds to a weakly-rotating tokamak with a high neutral edge density; in the second case, $\omega_{\mathrm{E}} / \omega_{\|} \sim \omega_{\|} / \nu \sim 1$. The latter case corresponds to a strongly-rotating tokamak which can have a poloidal Mach number $\mathrm{M}_{\mathrm{p}}=\left(\mathrm{u}_{\theta} / \mathrm{c}_{\mathrm{s}}\right)\left(\mathrm{B}_{\zeta} / \mathrm{B}_{\theta}\right)$ of order unity. We have shown that the ambipolarity condition takes different forms for the two cases, but the basic bifurcation exists in both limits.

The present calculation was partly motivated by the work of Cornelis et al., ${ }^{9}$ who showed that the experimental perpendicular conductivity on TEXTOR is dominated near the edge by ion-neutral friction [see their Figs. 11 and 12]. They also inferred that ionneutral friction contributes to the experimentally observed equilibrium bifurcation [see their Fig. 3]. Here, we have exhibited the neutral-dominated bifurcation analytically from a simple model.

This work may also be relevant to earlier studies of electrode-induced H-modes. For example, in Ref. $15 \mathrm{H}$-modes were produced with either sign of the electrode current and a sharp bifurcation was observed for the case $\mathrm{I}<0$ in the notation of the present paper. It was also noted that the required electrode voltages and currents were sensitive to the use of boronization, which may be related to changes in the neutral recycling.

More generally, this work is relevant to the recent interest in understanding how neutrals influence the plasma edge and the H-mode. The present model suggests that ionneutral charge exchange can influence the L-H transition in subtle ways by affecting the underlying ambipolarity constraint in the presence of a non-ambipolar drive due to external probes, internal turbulence, or other sources.

\section{Acknowledgements}

This work was supported by the U.S. Department of Energy (DOE) under DOE Grant No. DE-FG03-97ER54392; however, this support does not constitute an endorsement by the DOE of the views expressed herein. 


\section{References}

${ }^{1}$ T. Fukuda, M. Sato, T. Takizuka, K. Tsuchiya, Y. Kamada, H. Takenaga, and JT-60 Team, "H Mode Transition and Power Threshold in JT-60U," in Plasma Physics and Controlled Nuclear Fusion Research 1996, Proceedings of the $16^{\text {th }}$ International Conference, Montreal, 1996, (International Atomic Energy Agency, Vienna, 1997), Vol. 1, pp. 857-866.

${ }^{2}$ R. J. Groebner, T. S. Carlstrom, K. H. Burrell, S. Coda, E. J. Doyle, P. Gohil, K. W. Kim, R. Maingi, R. A. Moyer, Q. Peng, C. L. Rettig, T. L. Rhodes, D. P. Schissel, G. M. Staebler, R. D. Stambaugh, D. M. Thomas, and J. G. Watkins, "Study of H-mode Threshold Conditions in DIII-D," in Plasma Physics and Controlled Nuclear Fusion Research 1996, Proceedings of the $16^{\text {th }}$ International Conference, Montreal, 1996, (International Atomic Energy Agency, Vienna, 1997), Vol. 1, pp. 867-873.

${ }^{3}$ B. A. Carreras, L. W. Owen, R. Maingi, P. K. Mioduszewski, T. N. Carlstrom and R. J. Groebner, Phys. Plasmas 5, 2623 (1998).

${ }^{4}$ R. L. Boivin, J. A. Goetz, A. E. Hubbard, J. W. Hughes, I. H. Hutchinson, J. H. Irby, B. LaBombard, E. S. Marmar, D. Mossessian, C. S. Pitcher, J. L. Terry, B. A. Carreras, and L. W. Owen, Phys. Plasmas 7, 1919 (2000), and references therein.

${ }^{5}$ T. Fukuda, T. Takizuka, K. Tsuchiya, Y. Kamada, and N. Asakura, Plasma Phys. Contr. Fusion 42, A289 (2000).

${ }^{6}$ K. C. Shaing and E. C. Crume, Jr., Phys. Rev. Lett. 63, 2369 (1989).

${ }^{7}$ K. C. Shaing, E. C. Crume, Jr., and W. A. Houlberg, Phys. Fluids B 2, 1496 (1990).

${ }^{8}$ K. C. Shaing, A. Y. Aydemir, W. A. Houlberg, and M. C. Zarnstorff, Phys. Rev. Lett. 80, 5353 (1998).

${ }^{9}$ J. Cornelis, R. Sporken, G. Van Oost, and R. R. Weynants, Nucl. Fusion 34, 171 (1994);

${ }^{10}$ K. C. Shaing and C. T. Hsu, Phys. Plasmas 2, 1801 (1995).

${ }^{11}$ A. H. Boozer, Phys. Fluids 19, 149 (1976).

${ }^{12}$ P. J. Catto, P. Helander, J. W. Connor, and R. D. Hazeltine, Phys. Plasmas 5, 3961 (1998).

${ }^{13}$ A. Hassam, T. Antonsen, J. Drake, and C. S. Liu, Phys. Rev. Lett. 66, 312 (1991).

${ }^{14}$ A. B. Hassam and J. F. Drake, Phys. Fluids B 5, 4022 (1993).

${ }^{15}$ R. R. Weynants, G. Van Oost, G. Bertschinger, J. Boedo, et al., Nucl. Fusion 32, 837 (1992). 


\section{Figure Captions}

Fig. 1 Plot of I in Eq. (41) vs $\xi$ for $q=4$ and (a) $\xi_{d}=0$ and (b) $\xi_{d}=1$.

Fig. 2 Plot of solution boundaries in $\left(\xi_{\mathrm{d}}, \mathrm{q}\right)$ space for case 1.

Fig. 3 Plot of I in Eq. (42) vs $\xi$ for $q=4$ and $\delta=1$. 
(a)

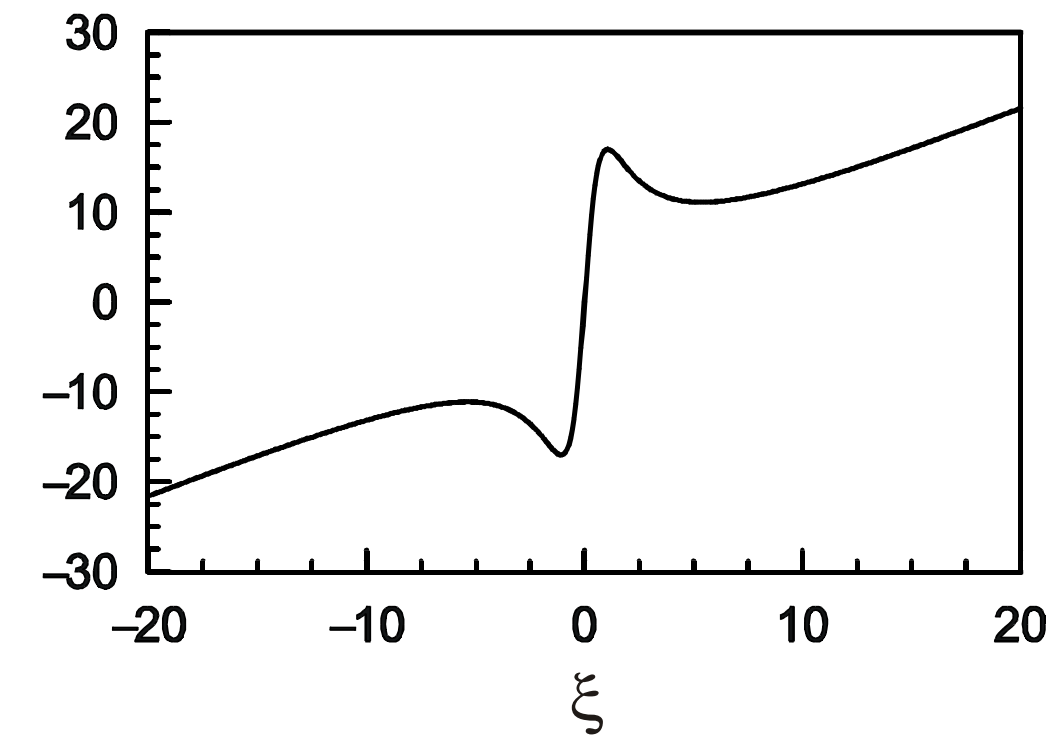

(b)

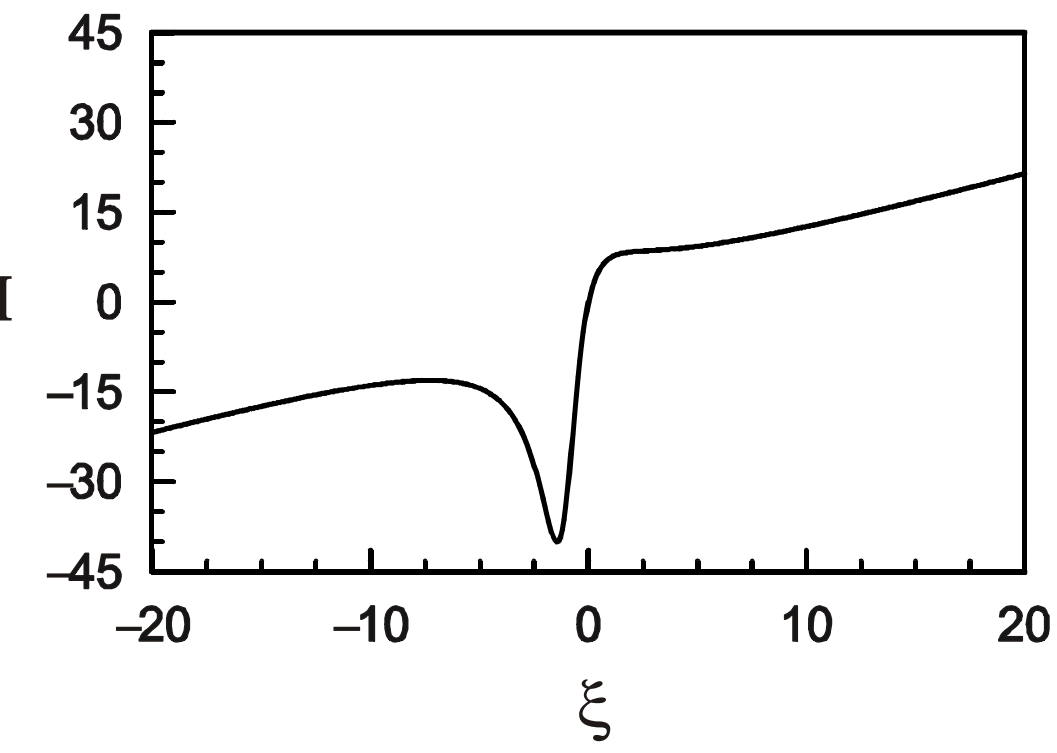

Fig. 1 Plot of I in Eq. (41) vs $\xi$ for $\mathrm{q}=4$ and (a) $\xi_{\mathrm{d}}=0$ and (b) $\xi_{\mathrm{d}}=1$. 


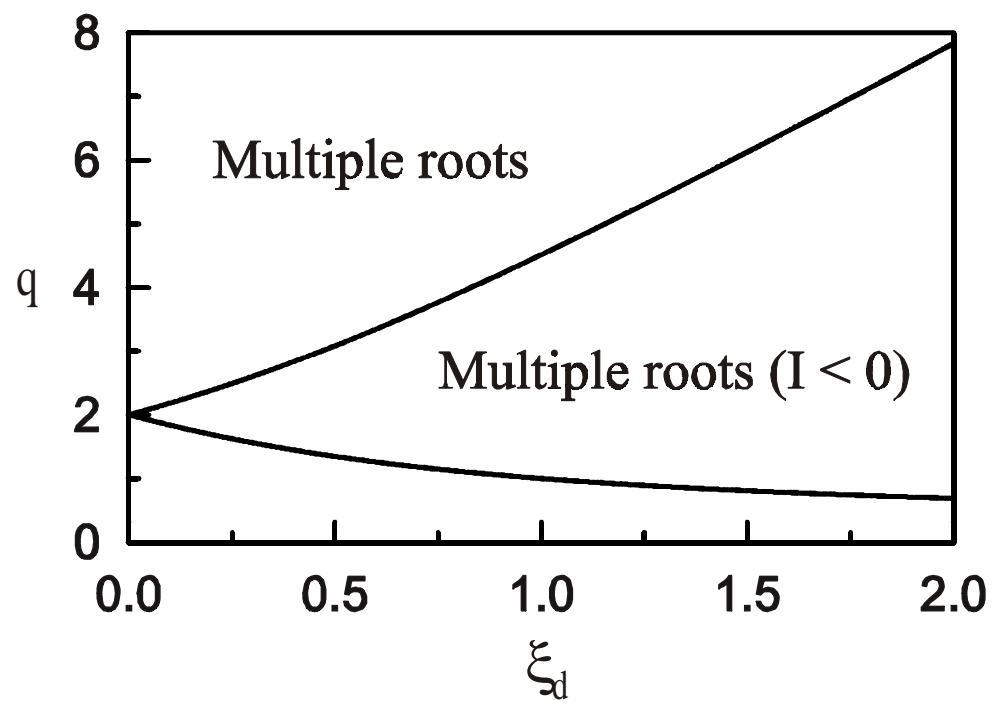

Fig. 2 Plot of solution boundaries in $\left(\xi_{d}, q\right)$ space for case 1. 


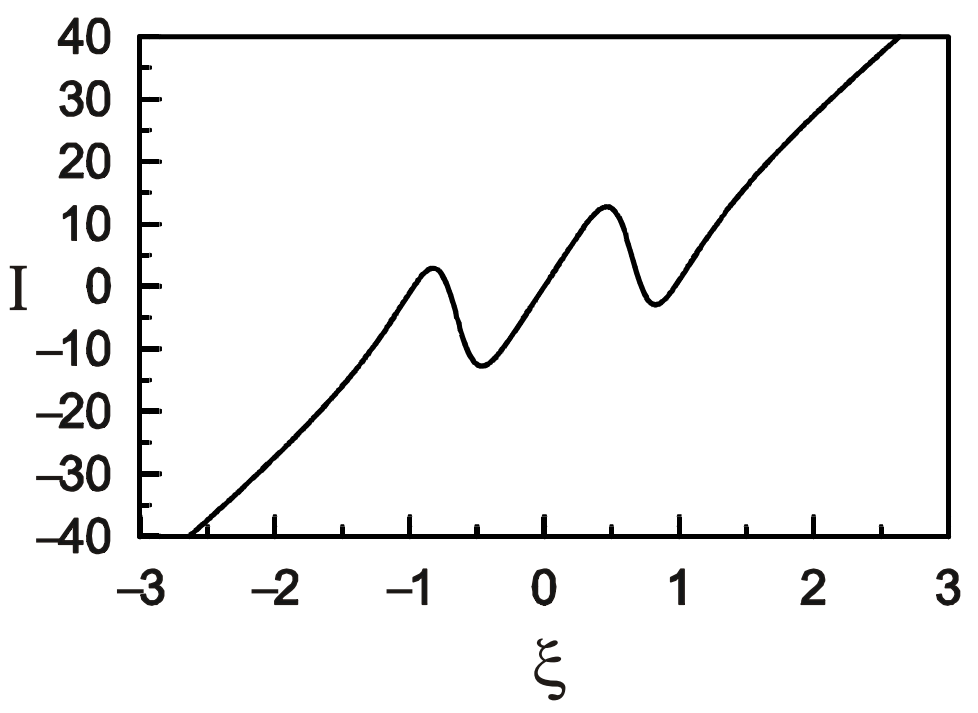

Fig. 3 Plot of I in Eq. (42) vs $\xi$ for $q=4$ and $\delta=1$. 